ISSN 2075-4701

www.mdpi.com/journal/metals/

Article

\title{
Nanometer-Scale Heterogeneities of the Structure of Zirconium-Based Bulk Metallic Glasses
}

\author{
Alexander S. Bakai, Evgenij V. Sadanov, Vjacheslav A. Ksenofontov, Sergej A. Bakai, \\ Julia A. Gordienko and Igor M. Mikhailovskij * \\ National Science Center "Kharkov Institute of Physics \& Technology”, Akademicheskaya 1, \\ 61108 Kharkov, Ukraine; E-Mails: bakai@kipt.kharkov.ua (A.S.B.); \\ sadanov.alexandr@mail.ru (E.V.S.); ksenofontov@kipt.kharkov.ua (V.A.K.); \\ serg.bakai@kipt.kharkov.ua (S.A.B.); admiral@kipt.kharkov.ua (J.A.G.) \\ * Author to whom correspondence should be addressed; E-Mail: mikhailovskij@kipt.kharkov.ua; \\ Tel.: +380-57-700-26-76; Fax: +380-57-335-36-86.
}

Received: 10 October 2012; in revised form: 26 October 2012 / Accepted: 29 October 2012 / Published: 15 November 2012

\begin{abstract}
Structure of amorphous alloys $\mathrm{ZrTiCuNiBe}$ and $\mathrm{ZrTiCuNiAl}$ is studied by means of low-field ion and combined field-emission microscopy. In both alloys the structural heterogeneities of nanometer-scale are clearly revealed. The surface layers formed by field evaporation possess a cellular structure. The cells have polygonal shape with transverse size ranging from $2 \mathrm{~nm}$ to $20 \mathrm{~nm}$. It is established that variance of the local energy of field evaporation is of $0 \%-5 \%$ in the cell body. A local minimum of the field evaporation energy is observed within the cell boundaries (intercluster boundaries). In the minimum the depth is measured to be of $0.8 \mathrm{eV}$.
\end{abstract}

Keywords: bulk metallic glasses; field ion microscopy; nanostructure; field emission; clusters

\section{Introduction}

The problem of structure-properties connection is not solved yet for metallic glasses (MG), in part for bulk metallic glasses (BMGs), mostly because a clear understanding of their atomic structure is still questionable [1-3]. In spite of the fact the structure translational invariance is absent in metallic glasses, a short- and medium-range order in form of the regular arrangement of adjacent polyhedra is established [4-10], providing a basis for extending a structural description beyond the nearest neighbor 
shell. According to the polycluster model [5-7] of amorphous solids, metallic glasses consist of locally regular clusters, whose atomic structure can be incompatible with the translational invariance. Existence of narrow topologically disordered intercluster boundaries in polyclusters is a logical corollary of this model. For this reason, the dislocationless slip at intercluster boundaries, formation and motion of dislocationlike defects are the main mechanism of the inhomogeneous plastic deformation of the polycluster MG. As a consequence, strain-induced softening of MG at deformation (unlike to the typically observed hardening of deformed crystalline metals) is explained under assumption that MG is consisting of "hard" amorphous grains (clusters) surrounded by soft intercluster boundaries, similarly to nanocrystalline materials [11-13]. A functional form for deformation-induced softening is obtained assuming that the dislocationless slip at intercluster boundaries is the main mode of the low-temperature plastic deformation [7].

Worth to note is that idea on the structural heterogeneities of glasses (more ordered amorphous grains surrounded by soft intercluster boundaries was already formulated by Lebedev at the first half of the past century [5] and was reincarnated in many publications. One of the recent papers devoted to this problem is [14]. Nevertheless, structural features of the intercluster layers are still not studied properly and debated. While in the covalently bonded glasses they are believed to be continuously disordered layers [5], in the metallic glasses they are assumed to be topologically disordered intercluster boundaries with structure similar to that of large angle boundaries in crystals. One can expect that in the multicomponent BMGs compositionally differing from the cluster body amorphous boundary layers are forming along with existence of the narrow topologically disordered boundaries. In this case compositional heterogeneities are accompanying the topological disorder.

Presence of inner boundaries in MG essentially impacts diffusional transport, crystallization kinetics, electric resistivity, phonon scattering and localization, low-temperature anomalies of specific heat capacity and thermal conductivity, etc.

In this paper, the combined methods of field emission microscopy (FEM) in the field ion and electron modes and low-field ion microscopy (LFIM) are applied for investigation of the fine structure of the $\mathrm{Zr}_{41} \mathrm{Ti}_{14} \mathrm{Cu}_{12.5} \mathrm{Ni}_{10} \mathrm{Be}_{22.5}$ and $\mathrm{Zr}_{52.5} \mathrm{Ti}_{5} \mathrm{Cu}_{17.9} \mathrm{Ni}_{14.6} \mathrm{Al}_{10}$ BMGs. The investigations are focused on finding of the intercluster boundaries and studies of their features.

\section{Results and Discussion}

A typical FIM image of a $\mathrm{Zr}_{41} \mathrm{Ti}_{14} \mathrm{Cu}_{12.5} \mathrm{Ni}_{10} \mathrm{Be}_{22.5}$ alloy formed by low-temperature field evaporation in high vacuum to a depth of about $50 \mathrm{~nm}$ is shown in Figure 1. The FIM images of this alloy are characterized by an isotropic uncorrelated distribution of bright emission centers. Each of the emission centers corresponds to an isolated surface atom or a small group of atoms. In both investigated zirconium-based BMGs no compositional peculiarities of the structure were found in the FIM images. 
Figure 1. The FIM image of a $\mathrm{Zr}_{41} \mathrm{Ti}_{14} \mathrm{Cu}_{12.5} \mathrm{Ni}_{10} \mathrm{Be}_{22.5}$ alloy formed by low-temperature field evaporation in high vacuum to a depth of about $50 \mathrm{~nm}$. The scale bar is $5 \mathrm{~nm}$.

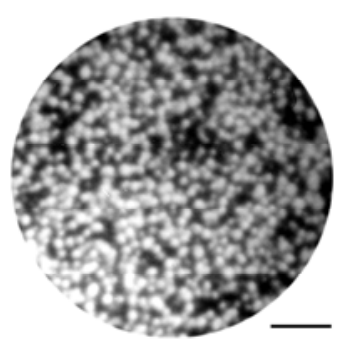

In Figure 2 typical LFIM images of $\mathrm{Zr}_{41} \mathrm{Ti}_{14} \mathrm{Cu}_{12.5} \mathrm{Ni}_{10} \mathrm{Be}_{22.5} \mathrm{BMG}$ at $17.0 \mathrm{kV}$ (a) and $18.5 \mathrm{kV}$ (b) are shown. The LFIM image in Figure $2 \mathrm{~b}$ was obtained after additional field evaporation of the surface layer of thickness up to $100 \mathrm{~nm}$. In the both images the nanometer-scale heterogeneities of the structure of $\mathrm{Zr}_{41} \mathrm{Ti}_{14} \mathrm{Cu}_{12.5} \mathrm{Ni}_{10} \mathrm{Be}_{22.5} \mathrm{BMG}$ are clearly seen. The morphology of these surface layers is characterized by a cellular structure. The cells had an irregular elongated polygonal shape with transverse dimensions ranging from $2 \mathrm{~nm}$ to $20 \mathrm{~nm}$. The mean diameter of the surface cell, stereologically determined by counting of the number of intersections of a line of known length with (Figure 1) boundaries [15], were $7 \pm 2 \mathrm{~nm}$ and $14.9 \pm 4 \mathrm{~nm}$ in the small and large cross-sections, respectively. The stereological relationships are based on assumption that the cell structure is isotropic, uniform and random so that all portions of the structure are equally represented.

Figure 2. LFIM images of $\mathrm{Zr}_{41} \mathrm{Ti}_{14} \mathrm{Cu}_{12.5} \mathrm{Ni}_{10} \mathrm{Be}_{22.5} \mathrm{BMG}$ at (a) $17.0 \mathrm{kV}$ and (b) $18.5 \mathrm{kV}$. The cellular morphology and nanometer-scale heterogeneities of the structure of are observed. Scale bar is $20 \mathrm{~nm}$.

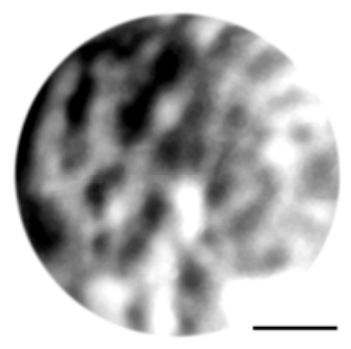

(a)

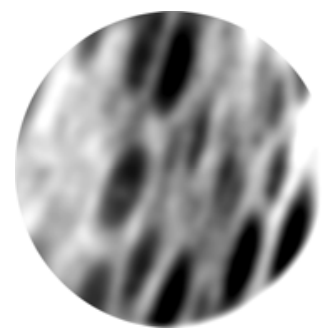

(b)

The LFIM images of $\mathrm{Zr}_{41} \mathrm{Ti}_{14} \mathrm{Cu}_{12.5} \mathrm{Ni}_{10} \mathrm{Be}_{22.5} \mathrm{BMG}$ show that the alloy is locally anisotropic. The level of statistical significance for locally anisotropic structures is larger for the dimensionless ratio of mean diameters of the surface cells in the small and large cross-sections. This aspect ratio of the cells in the LFIM images of this alloy (Figure 2) reaches the value of 2.1.

The LFIM images of $\mathrm{Zr}_{52.5} \mathrm{Ti}_{5} \mathrm{Cu}_{17.9} \mathrm{Ni}_{14.6} \mathrm{Al}_{10}$ BMG also clearly revealed the nanometer-scale cellular topographic heterogeneities (Figure 3). The cells have an irregular polygonal shape with transverse dimensions ranging from $3 \mathrm{~nm}$ to $15 \mathrm{~nm}$. However, unlike to the $\mathrm{Zr}_{41} \mathrm{Ti}_{14} \mathrm{Cu}_{12.5} \mathrm{Ni}_{10} \mathrm{Be}_{22.5}$ $\mathrm{BMG}$, the cell structure is isotropic and the mean diameter of the surface cells is $9.2 \pm 2 \mathrm{~nm}$ in all directions on the surface. This value is obtained by stereological analysis of several cross-sections normal to specimen axis during the process of chemically induced field evaporation, two of which are presented in Figure 3. 
Figure 3. Typical LFIM images of $\mathrm{Zr}_{52.5} \mathrm{Ti}_{5} \mathrm{Cu}_{17.9} \mathrm{Ni}_{14.6} \mathrm{Al}_{10} \mathrm{BMG}$ (a) before and (b) after chemically induced field evaporation at a depth of about $100 \mathrm{~nm}$. Scale bar is $10 \mathrm{~nm}$.

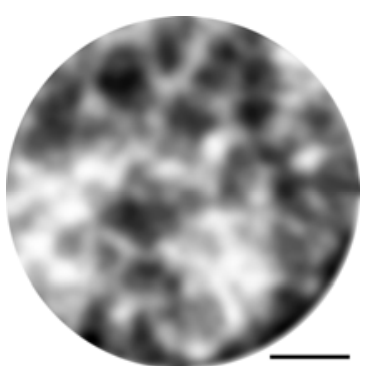

(a)

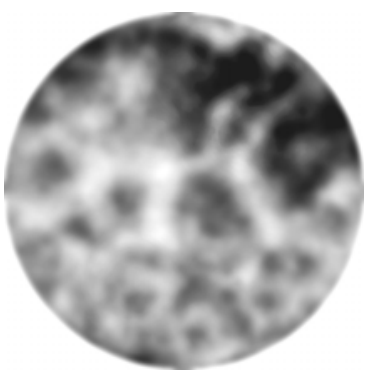

(b)

Field electron images of both BMGs also reveal substantial nanometer-scale heterogeneities of field emission from the surface formed by low-temperature field evaporation in high vacuum at 53-77 $\mathrm{K}$ (Figure 4a). The contour plot of constant brightness levels of the image is presented in Figure $4 \mathrm{~b}$. Difference between local energies of field evaporation in sample sites, which correspond to the neighbouring numbered contour lines of constant brightness in Figure $4 \mathrm{~b}$, is equal to $\Delta Q_{n} / Q_{n}^{\max }=(1.0 \pm 0.2) \times 10^{-2}$. Distances between centers of increased brightness in the investigated samples are changing within the range $5-20 \mathrm{~nm}$.

Figure 4. (a) Field electron image and (b) contour diagram of constant brightness levels for the $\mathrm{Zr}_{41} \mathrm{Ti}_{14} \mathrm{Cu}_{12.5} \mathrm{Ni}_{10} \mathrm{Be}_{22.5}$ BMG. An intercluster boundary is shown by arrow. Scale bar is $5 \mathrm{~nm}$.

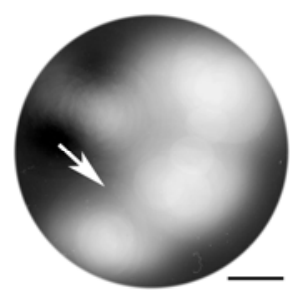

(a)

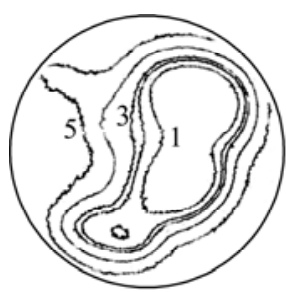

(b)

The method of field emission microscopy allows determining local values of field evaporation energy $Q_{n}$. In a model of the image forces [16]

$$
Q_{n}=\frac{1}{2}\left(\frac{n^{3} e^{3} F}{\pi \varepsilon_{0}}\right)^{1 / 2}
$$

where $n$ is the multiplicity of ionization of the evaporated atoms; $e$ is the electron charge. Variation of field evaporation energy was determined by shifts of contours of constant brightness levels in the field Figure 2. From Fowler-Nordheim relationship [17] for a field emission one can directly derive

$$
\mathrm{d} j_{e} / j_{e}=\chi \mathrm{d} F / F
$$

where $\chi$ is the coefficient of the field sensitivity of electronic emission; $F$ is the local intensity of a field emission. In a mode of electron emission the field, $F$ changed within the range $2-4 \mathrm{~V} \mathrm{~nm}^{-1}$ and the value of $\chi$ was in the range of $14-20[6,18]$. 
Heterogeneity of the local evaporation field strength distribution in the mode of electron emission is caused by peculiarities of the surface microrelief formed at field evaporation in the field ion mode. It reflects heterogeneity of the field evaporation energy determined by Equation 1. Thus, the contour lines of constant brightness for electron emission image correspond to the evaporation energy isolines. Calculation of the absolute values of field evaporation energy $Q_{n}$ is complicate due to complexity of the charge spectrum of ions in a multicomponent alloy. But deviations of evaporation energy from the maximum value $Q_{n}^{\max }$ can be calculated directly using Equations 1 and 2 . The energy values shown in Figure 4 correspond to the difference of field evaporation energy, $\Delta Q_{0}$, in the cluster center and at isoenergetic contours, calculated from Equation 1 for the series of evaporation voltages.

According to these observations, variance of the local energy of field evaporation is $\sim 5 \%$ within cells of characteristic scale $\sim 10 \mathrm{~nm}$. The energy of evaporation decreases towards the cluster periphery, reaching a minimum value at the intercluster triple junctions (with $\Delta Q_{0}$ up to $1.30 \mathrm{eV}$ ). A local minimum energy, presumably at the intercluster boundary corresponds to $\Delta Q_{0}=0.8 \mathrm{eV}$.

The mean width of such boundaries determined by a local decrease in brightness due to the effect of preferred field evaporation is $2.5 \pm 1.0 \mathrm{~nm}$. This value is rather higher than that generally determined by the FIM method of field etching [6,19]. Obviously, one of the reasons for this difference may be relatively low lateral resolution of the FEM in the electron mode. The FEM pattern on the screen is not very sharp, because emitted electrons have a transverse velocity, which results in a scattering disk on the phosphor screen. The resolution limit of the FEM can be characterized by a parameter $\delta$, which is defined as the minimal diameter of the image disk divided by the image magnification. There is a general trend toward an increase of the FEM and FIM spatial resolution $\delta$ by miniaturization of pointed specimens $[16,20]$. Most of the calculations of this quantity were performed in the micron and submicron ranges of the specimen lateral dimension. The dependence of $\delta$ on the curvature radius of the top of needlelike specimen $\rho$ in the nanometer-scale range can be calculated using the following formula [20]:

$$
\delta=\sqrt{\sqrt{\frac{2}{m_{e} e V}} \cdot \hbar k r+\frac{4 \hbar F k^{2} r^{2}}{\sqrt{2 m_{e} \varphi} V}}
$$

with elementary charge $e$, electron mass $m_{e}$, Planck constant $\hbar$, image compression factor $k$, emitter radius of curvature $r$, potential $V$ and work function $\varphi$. Typically $k \approx 1.5$. With that Equation 3 gives estimation of the FEM resolution $\delta \approx 1.1-1.5 \mathrm{~nm}$. This value is considerably larger than that for a FIM $(0.3 \mathrm{~nm})[16]$ and may be responsible for the broadening of the intercluster boundary image.

Performed analysis of the evaporation field distribution on the surface of partially evaporated samples clearly show presence of inner interfaces (intercluster boundaries) and their triple joints in the BMGs investigated. The used tools do not allow to investigate details of microscopic structure of the interfaces including the local toplogical and compositional order but our studies allow to estimate, at least qualitatively, the role of the topological and compositional order in formation of the interfaces. The width of the boundary layer, in which the field evaporation energy is steeply decreasing, is not small, up to $2.5 \mathrm{~nm}$, as it is seen from the contour diagrams (Figure 4). This feature can be attributed to gradual changes of the composition in the boundary layer. Decomposition of $\mathrm{Zr}_{41} \mathrm{Ti}_{14} \mathrm{Cu}_{12.5} \mathrm{Ni}_{10} \mathrm{Be}_{22.5}$ glass and two-phase (Ti-reach and Be-reach) glass formation as result of liquid state aging (at $T>T_{\mathrm{g}}$ ) 
was revealed in $[21,22]$. Therefore compositional stratification of this glass is an expected property. Even without special aging at $T>T_{\mathrm{g}}$, partial decomposition of the glass occurs during the glass transition when solid-like amorphous germs separated by a liquid-like substance of a different composition are forming.

From the other hand, the measured minimal value of the field evaporation energy in the boundary layer $\left(\Delta Q_{0} \approx 0.8 \mathrm{eV}\right)$ is comparable with decrease of the atom binding energy within the disordered boundaries in polycrystals [23]. Of this evidence, one can conclude that a narrow topologically disordered intercluster boundary exists at the $\Delta Q_{0}$ maximum location. Apparently this boundary appears when the solid clusters (with compositionally inhomogeneous periphery) are contacting and the structure relaxation processes are frozen because of low temperature. It can be assumed that the peculiarities of the revealed heterogeneities of the field evaporation energy are evidence of narrow topologically disordered intercluster boundaries surrounded by compositionally inhomogeneous layers.

\section{Experimental Procedure}

Measurements of the nanometer-scale structural heterogeneities of zirconium based bulk metallic glasses were performed using a two-chamber field ion microscope (FIM) at the specimens' temperature 53-77 K [24]. Image was formed by using neon under a pressure of $2 \times 10^{-3}-2 \times 10^{-2} \mathrm{~Pa}$. The working chamber of the microscope was evacuated by cryogenic pumps to a residual gas pressure of $10^{-6} \mathrm{~Pa}$. In order to use the microscope in both the field electron and ion modes, a constant positive voltage of 3-25 kV, and alternating voltage of amplitude 4-28 kV and frequency $50 \mathrm{~Hz}$ was supplied to specimens. The total voltage supplied to the specimen was of the pulse type with the ratio of the amplitudes of positive and negative voltages varied from 6 to 10. The negative voltage pulses of duration $(2-3) \times 10^{-3} \mathrm{~s}$ was sufficient for creating on the specimen tip a field strength required for an electron current density of $10^{2}-10^{3} \mathrm{~A} \mathrm{~cm}^{-2}$.

Amorphous rods with a diameter of $12 \mathrm{~mm}$ and $50 \mathrm{~mm}$ length were smelted in a sealed quartz pipe in a pure argon atmosphere with subsequent quenching in water. By X-ray and electronic difractometry it was shown that the obtained material was completely amorphous and microscopically homogeneous. Thin disks $0.2 \mathrm{~mm}$ thick were cut from the rods. Then columns with sizes $10 \mathrm{~mm} \times 0.2 \mathrm{~mm} \times 0.2 \mathrm{~mm}$ were cut with the help of a diamond wire saw. Needlelike specimens with a curvature of 10-100 $\mathrm{nm}$ at the top were prepared by electrochemical etching at a constant voltage 3-10 V in a $15 \%$ solution of chloric acid in ethyl alcohol at room temperature.

The surface of the specimens after mounting in the microscope was cleaned by field desorption [16]. Then formation of the surface was carried out at 53-77 $\mathrm{K}$ by field evaporation to a depth not less than $10 \mathrm{~nm}$. Thus not only adsorbed layers and surface layers contaminated during electrochemical processing were removed, but also a specific surface microtopography of samples was created.

The prepared surface relief represents the distribution of local threshold values of evaporation field strength $F_{e}$. The observed contrast of FEM images is determined mainly by this distributions formed during the process of preliminary surface polishing by field evaporation. The value of $F_{e}$ was determined by comparison with the threshold field strength of ionization for atoms of imaging gas. The contour configuration for constant values of current density was determined by means of photometry with use of computer processing of brightness distribution in the field emission image. 
The structure of the amorphous alloys was also studied by means of low-field ion microscopy using the phenomenon of field etching of the specimen surface under the action of water vapor accompanied by the emission of positive ions in electric fields of $2 \mathrm{~V} \mathrm{~nm}^{-1}$ [25]. Consecutive dissociation of water molecules with the formation (in the intermediate state) of the $\mathrm{OH}$-radical bound to the metal surface followed by the formation of the oxide subsequently removed by the electric field was used to get specific FIM images. The products of chemical reactions occurring at the specimen surface during field etching ensure the formation of low-field ion images. Experiments were performed using the same FIM with the samples at room temperatures. The required concentration of water vapor in the chamber was attained by evacuation to a residual gas pressure of $10^{-1} \mathrm{~Pa}$. Since the image is formed by the flows of the material being evaporated and the products of chemical reactions occurring at the surface of the metal, this regime is dynamic and is accompanied by a continuous increase in the radius of the tip of the point sample. Gradual evaporation of micrometer-size samples to a large depth with continuous image recording forms the basis of the 3D nanometallography of BMGs.

\section{Conclusions}

Based on the obtained results, the following conclusions can be drawn:

The structures of the amorphous alloys ZrTiCuNiBe and ZrTiCuNiAl in the as-quenched state are characterized by the clearly revealed nanometer-scale structural heterogeneities.

The microtopography of these alloys formed by low-temperature field evaporation is characterized by a polygonal cellular structure. The LFIM images of $\mathrm{Zr}_{41} \mathrm{Ti}_{14} \mathrm{Cu}_{12.5} \mathrm{Ni}_{10} \mathrm{Be}_{22.5}$ BMG show that its cellular structure is anisotropic with aspect ratio of the cells $\sim 2.1$. The LFIM images of $\mathrm{Zr}_{52.5} \mathrm{Ti}_{5} \mathrm{Cu}_{17.9} \mathrm{Ni}_{14.6} \mathrm{Al}_{10}$ BMG also reveal the cellular heterogeneities with a scale of the order of $10 \mathrm{~nm}$. However, unlike to the $\mathrm{Zr}_{41} \mathrm{Ti}_{14} \mathrm{Cu}_{12.5} \mathrm{Ni}_{10} \mathrm{Be}_{22.5} \mathrm{BMG}$, the cell structure is isotropic.

The isoenergetic cross-sections of revealed nanometer-scale heterogeneities and contour diagrams of energy isolines were determined for both of these alloys. According to this analysis, deviations of a local energy of field evaporation were within the range $\sim 5 \%$ with a local minimum energy, presumably at the intercluster boundary, corresponding to $\Delta Q_{0} \approx 0.8 \mathrm{eV}$. The mean width of such boundaries determined by a local decrease in brightness due to the effect of preferred field evaporation is $2.5 \pm 0.5 \mathrm{~nm}$. The observed heterogeneities of the field emission contrast and local energy of evaporation are interpreted as evidence of narrow the topologically disordered intercluster boundaries surrounded by compositionally inhomogeneous layers.

\section{Acknowledgments}

We thank N. Wanderka for discussions and comments. This work was supported by the Ukraine State Program "Nano-Technologies and Nano-Materials" through Project \# 1.1.1.48.

\section{Conflict of Interest}

The authors declare no conflict of interest. 


\section{References}

1. Okamura, A.H.; Inoue, A.; Masumoto, T. Decomposition and primary crystallization in undercooled $\mathrm{Zr}_{41.2} \mathrm{Ti}_{13.8} \mathrm{Cu}_{12.5} \mathrm{Ni}_{10.0} \mathrm{Be}_{22.5}$ melts. Acta Metall. Mater. 1993, 41, 915-917.

2. Peker, A.; Johnson, W.L. A highly processable metallic glass: $\mathrm{Zr}_{41.2} \mathrm{Ti}_{13.8} \mathrm{Cu}_{12.5} \mathrm{Ni}_{100} \mathrm{Be}_{22.5}$. Appl. Phys. Lett. 1993, 63, 2342-2344.

3. Du, X.; Huang, J.C.; Hsieh, K.-C.; Jang, J.S.C.; Liaw, P.K.; Chen, H.-M.; Chou, H.-S.; Lai, Y.-H. Designing ductile Zr-based bulk metallic glasses with phase separated microstructure. Adv. Eng. Mater. 2009, 11, 387-391.

4. Gaskell, P.H. In Glassy Metals II; Beck, H., Guentherodt, H.-J., Eds.; Topics in Applied Physics; Springer: Berlin, Germany, 1994; Volume 53, pp. 5-29.

5. Bakai, A.S. The Polycluster Concept of Amorphous Solids. In Glassy Metals III; Beck, H., Guentherodt, H.-J., Eds.; Topics in Applied Physics; Springer: Heidelberg, Germany, 1994; Volume 72, pp. 208-255.

6. Bakai, A.S.; Mikhailovskij, I.M.; Mazilova, T.I.; Wanderka, N. Field emission microscopy of the cluster and subcluster structure of a $\mathrm{Zr}-\mathrm{Ti}-\mathrm{Cu}-\mathrm{Ni}-\mathrm{Be}$ bulk metallic glass. Low Temp. Phys. 2002, 28, 279-283.

7. Lazarev, N.; Bakai, A. Dislocationless sliding in a polycluster glass. Int. J. Mater. Res. 2011, 102, $1147-1151$.

8. Sheng, H.W.; Liu, H.Z.; Cheng, Y.Q.; Wen, J.; Lee, P.L.; Luo, W.K.; Shastri, S.D.; Ma, E. Polyamorphismin a metallic glass. Nat. Mater. 2007, 6, 192-197.

9. Miracle, D.B. A structural model for metallic glasses. Nat. Mater. 2004, 3, 697-702.

10. Miracle, D.B.; Harrowell, P. Noncrystalline compact packings of hard spheres of two sizes: Bipyramids and the geometry of common neighbors. J. Chem. Phys. 2009, 130, 114505.

11. Bei, H.; Xie, S.; George, E.P. Softening caused by profuse shear banding in a bulk metallic glass. Phys. Rev. Lett. 2006, 96, 105503.

12. Bakai, A.S.; Bakai, S.A.; Eckert, J.; Neklyudov, I.M.; Savchenko, V.I. Mixed viscous flow and softening of bulk metallic glasses. J. Non Cryst. Solids 2007, 353, 3754-3757.

13. Bakai, A.S.; Bakai, S.A.; Mikhailovskij, I.M.; Neklyudov, I.M.; Stoev, P.I.; Macht, M.-P. On the nature of the Kaiser effect in metallic glasses. JETP Lett. 2002, 76, 218-221.

14. Egami, T.; Poon, S.J.; Zhang, Z.; Keppens, V. Glass transition in metallic glasses: A microscopic model of topological fluctuations in the bonding network. Phys. Rev. 2007, 76, 024203.

15. Russ, J.C.; Dehoff, R.T. Practical Stereology, 2nd ed.; Plenum Press: New York, NY, USA, 2000.

16. Miller, M.K.; Cerezo, A.; Heatherington, M.G.; Smith, G.D.W. Atom Probe Field Ion Microscopy; Clarendon Press: Oxford, England, 1996.

17. Modinos, A. Field, Thermionic and Secondary Electron Emission; Plenum Press: New York, NY, USA, 1984.

18. Lazarev, B.G.; Ksenofontov, V.A.; Mikhailovskii, I.M.; Velikodnaya, O.A. Nanostructure of superconducting Nb-Ti alloys. Low Temp. Phys. 1998, 24, 205-209.

19. Bakai, A.S.; Kul'ko, V.V.; Mikhailovskij, I.M.; Rabukhin, V.B.; Velikodnaya, O.A. Field-emission microscopy of amorphous CoSi alloy. J. Non Cryst. Solids 1995, 182, 315-320. 
20. Heeres, E.C.; Oosterkamp, T.H.; de Jonge, N. Size of the localized electron emission sites on a closed multiwalled carbon nanotube. Phys. Rev. Lett. 2012, 108, 036804.

21. Macht, M.-P.; Wanderka, N.; Wiedenmann, A.; Wollenberger, H.; Wei, Q.; Fecht, H.J.; Klose, S.G. Decomposition of the supercooled liquid of the bulk amorphous alloy $\mathrm{Zr}_{41} \mathrm{Ti}_{14} \mathrm{Cu}_{12.5} \mathrm{Ni}_{10} \mathrm{Be}_{22.5}$. Mater. Sci. Forum 1996, 225-227, 65-70.

22. Bakai, A.S.; Wanderka, N.; Macht, M.-P.; Mikhailovskij, I.M.; Mazilova, T.I. Field-emission microscopy of a bulk amorphous alloy $\mathrm{Zr}_{41} \mathrm{Ti}_{14} \mathrm{Cu}_{12.5} \mathrm{Ni}_{10} \mathrm{Be}_{22.5}$. Met. Phys. Adv. Tech. 1999, 18, $445-450$.

23. Sutton, A.P.; Ballufi, R.W. Interfaces in Crystalline Materials; Clarendon: Oxford, England, 1995.

24. Neklyudov, I.M.; Sadanov, E.V.; Tolstolutskaja, G.D.; Ksenofontov, V.A.; Mazilova, T.I.; Mikhailovskij, I.M. Interstitial atoms in tungsten: Interaction with free surface and in situ determination of formation energy. Phys. Rev. 2008, 78, 115418.

25. Ksenofontov, V.A.; Sadanov, E.V.; Velikodnaya, O.A. Low-field ion microscopy. Tech. Phys. 2009, 54, 580-585.

(C) 2012 by the authors; licensee MDPI, Basel, Switzerland. This article is an open access article distributed under the terms and conditions of the Creative Commons Attribution license (http://creativecommons.org/licenses/by/3.0/). 